\title{
A Multistrip-MRPC prototype for the CBM Time-of-Flight wall
}

Ingo DEPPNER ${ }^{*}$ and Norbert Herrmann ${ }^{\dagger}$

Physikalisches Institut der Universitä̈ Heidelberg

E-mail: deppner@physi.uni-heidelberg.de

herrmann@physi.uni-heidelberg.de

In the future Compressed Baryonic Matter (CBM) experiment hadron identification is provided by a Time-of-Flight (ToF) wall of $120 \mathrm{~m}^{2}$ composed of Multigap Resistive Plate Chambers (MRPCs). The conceptual design foresees five rate depending concentric arranged regions named A to $\mathrm{E}$ where $\mathrm{A}$ is the area having fluxes below $1 \mathrm{kHz} / \mathrm{cm}^{2}$ in average. For this region A which covers approximate $55 \%$ of the total area we developed a Multistrip-MRPC containing float glass as resistive electrode material. Here we present the design of this prototype and in particular results obtained during in beam tests at GSI and COSY.

XI Workshop on Resistive Plate Chambers and Related Detectors

5-10 February, 2012

Laboratori Nazionali di Frascati dell'INFN - Frascati (Rome) - Italy

* Speaker.

${ }^{\dagger}$ Work supported by BMBF 06HD9121I and EU/FP7 I3 Hadron Physiks 2 


\section{Introduction}

The Compressed Baryonic Matter (CBM) spectrometer will be located at the Facility for Antiproton and Ion Research (FAIR) in Darmstadt, Germany. CBM aims to study strongly interacting matter under extreme conditions. Therefore it is designed as a multi-purpose device which will be able to measure hadrons, electrons and muons in heavy-ion collisions [1]. The charged hadron identification in particular the pion, kaon and proton separation up to a momentum of $4 \mathrm{GeV} / \mathrm{c}$ will be realized by measuring the Time-of-Flight (ToF) of these particles with an accuracy of 80 ps. In the current conceptual design a ToF-wall composed of Multigap Resistive Plate Chambers (MRPCs) with a total active area of $120 \mathrm{~m}^{2}$ located $10 \mathrm{~m}$ from the interaction point is considered [2]. UrQMD [3] simulation of gold on gold collisions at $25 \mathrm{AGeV}$ with $10 \mathrm{MHz}$ interaction rate show that $55 \%$ of the active area has in average per readout channel a particle flux below $1 \mathrm{kHz} / \mathrm{cm}^{2}$ (see fig. 1a). This low particle flux allows to use low priced float glass as resistive material for MRPC electrodes. In this paper/proceeding we report on a Multistrip-MRPC prototype for the low rate area called region A (see fig. 1b) developed at Physikalisches Institut in Heidelberg.

(a)

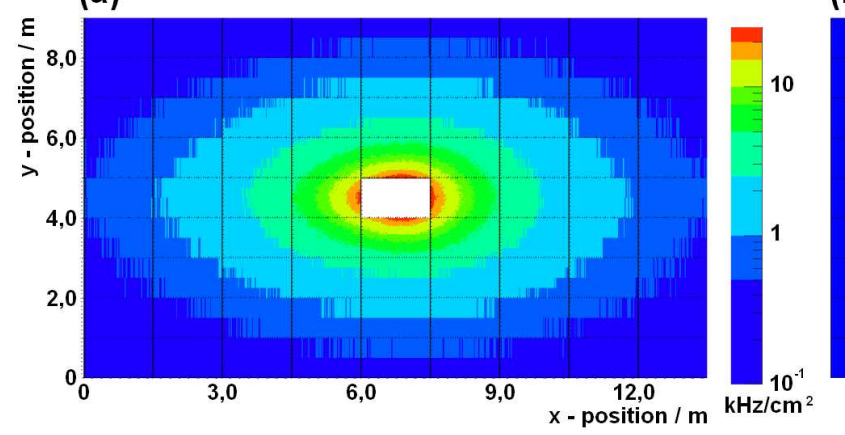

(b)

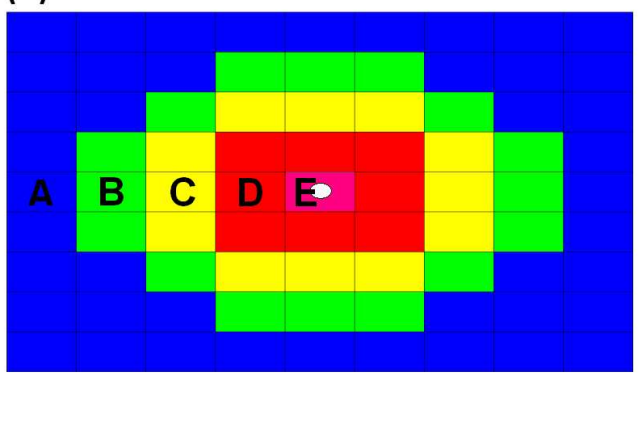

Figure 1: (a) charged particle flux distribution on the surface of the planed ToF-wall. The wall is subdivided in 80 super modules with $1.5 \mathrm{~m}^{2}$ each. (b) Tof-wall sectioning in 5 different regions named $\mathbf{A}$ to $\mathbf{E}$ depending on particle flux. The region A represent the low rate region $\left(\leq 1 \mathrm{kHz} / \mathrm{cm}^{2}\right)$ and covers $55 \%$ of the total area.

\section{Design of the MMRPC prototype}

In order to approach a real size demonstrator (approx. $50 \mathrm{~cm} \times 50 \mathrm{~cm}$ ) for region A a fully symmetric MRPC prototype with a active area of $32 \times 27 \mathrm{~cm}^{2}$ was developed. Its active volume is subdivided by nine $0.5 \mathrm{~mm}$ thick float glass plates. The gap between the glass plates is ensured by $220 \mu \mathrm{m}$ thick fishing lines. Two Kapton ${ }^{\circledR}$ foils coated on one side with a conductive material (surface resistivity $\approx 200 \mathrm{M} \Omega / \square$ ) are placed with the coating on top of the glass stack. They serve as high voltage electrodes and ensure in the same time the insolation to the signal pick-up electrodes. The pick-up electrode (1.6 mm PCB) contains 32 readout strips with a strip to gap pitch of $7 \mathrm{~mm}$ to $3 \mathrm{~mm}$ respectively (see fig.2). The advantage of the proposed strip configuration is that the strip width is comparable to the mean cluster size of an avalanche which was shown to be approximately $7 \mathrm{~mm}$ at a field of $108 \mathrm{kV} / \mathrm{cm}$ [4]. In addition this strip configuration together with the total number of gaps is optimized to realize an impedance of $100 \Omega$ in order to match the input impedance of the front end electronic (FEE). Furthermore the signal transition between strip and 
FEE is also optimized concerning minimization of signal reflections. High frequency reflectometer measurement show an impedance of $93 \Omega$. This prototype is build in such a way that one can connect alternatively a twisted pair cable or the FEE-cards directly to the RPC pick-up electrode (see fig. 3a). Therefore RPC signals which are in the order of $20 \mathrm{mV}$ can be discriminated immediately without quality loss due to propagation in the cable. During in beam tests both possibilities were tested and compared.

(a)

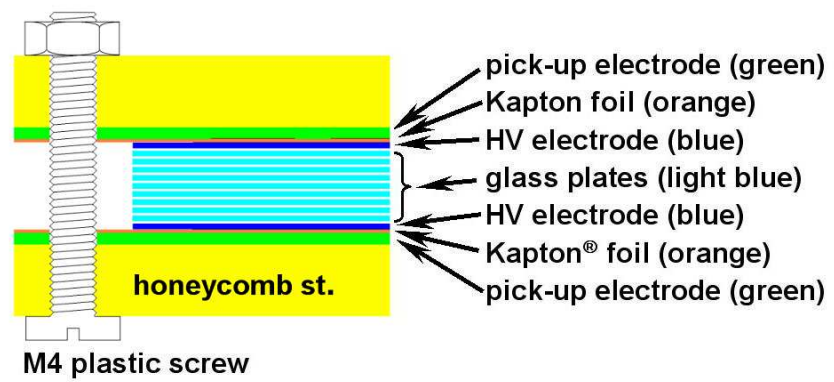

(b)

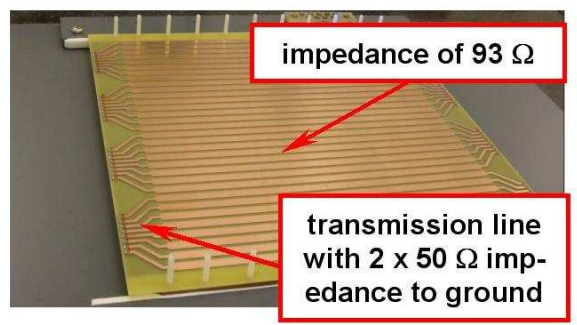

Figure 2: (a) cross section of the fully differential MMRPC. Honeycomb structured plates ensure the mechanical stability. (b) signal pick-up electrode. Special effort regarding impedance matching was investigated.

\section{Results}

With this prototype two in-beam test were performed. One with pions at beam momentum of $1.7 \mathrm{GeV} / \mathrm{c}$ at GSI Helmholtzzentrum für Schwerionenforschung in Darmstadt and a second test at the Cooler Synchrotron (COSY) at Forschungszentrum Jülich with protons having a momentum of $3.35 \mathrm{GeV} / \mathrm{c}$. Figure $3 \mathrm{~b}$ shows exemplary the experimental setup as used in the test experiment at COSY.

(a)

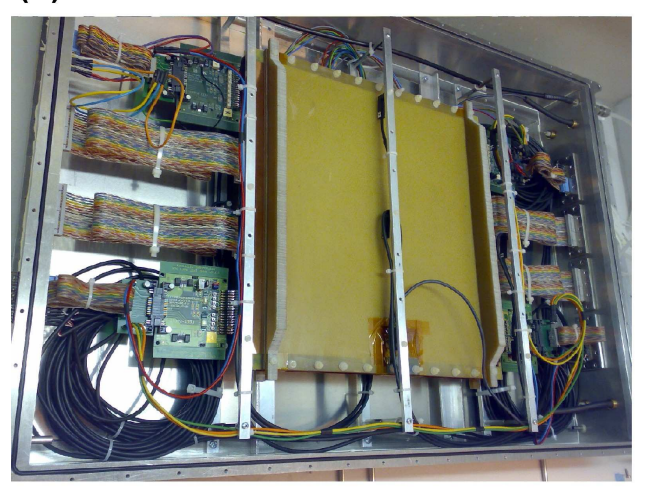

(b)

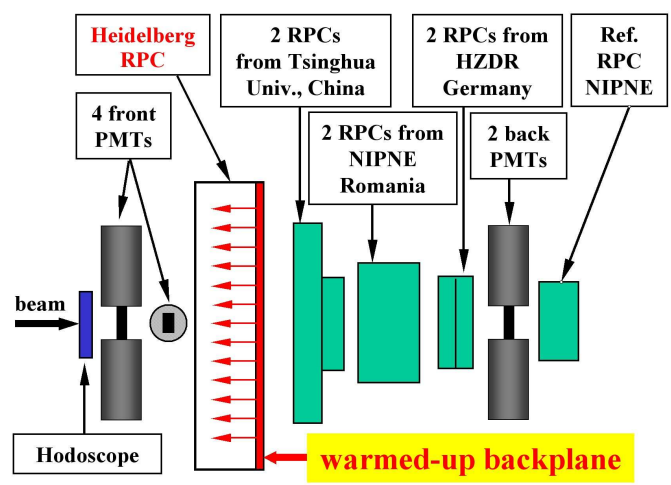

Figure 3: (a) MMRPC mounted in a aluminum box. 16 out of 32 readout strips were equipped with FEEboards directly on the counter. (b) Test setup installed at. The hodoscope delivers information about the beam profile. The back plane of the Heidelberg-RPC box was heatable. 
The trigger was formed by coincidence between the OR of the two front plastic scintillators read out by two photomultiplier tubes (PMT) each and the plastic scintillator in the back of the setup. For the analysis a software coincidence of all 6 PMTs was requested. The two front scintillators were used due to a better performance as reference for timing measurements. The split signals of the PMTs were discriminated by a constant fraction discriminator and fed into a CAEN V1290N TDC. The remaining analog signal was digitized in a LeCroy QDC. The MRPC was connected to custom designed preamplifier cards called PADI [5]. In order to extract the time over threshold information the LVDS signals from PADI output were split and fed into two different TDCs (Caen V1290A). One of them measured the rising edge and the other the falling edge of the signal. This is necessary because the width of the signal is smaller than the edge detection capability of this type of TDC. All TDCs were synchronized by a external $40 \mathrm{MHz}$ clock. Additional information about the beam position and profile was delivered by a hodoscope mounted in front of the scintillator (see fig. $3 b$ ). The granularity of this device is $1 \mathrm{~mm}^{2}$. During this test it was possible to warm up the back plane of the gas box. Inside the box the RPC was surrounded by 18 PT100 temperarure sensors. Therefore a full temperature control was warranted.

\section{Efficiency}

The efficiency of the RPC is obtained by comparing the total number of events in the coincidence of all PMTs with the number of hits in the RPC itself. Figure 4a shows the obtained dependence of the efficiency on the applied RPC high voltage (RPC-HV). The red squares symbolize the measured efficiency with the electronics attached directly to the RPC inside the box. The threshold of PADI which is applied after the amplification (Gain $\approx 8$ ) of the analog signal was set remotely to $27 \mathrm{mV}$. The black data points are related to the results obtained with the electronics located outside the chamber. The threshold in this cases was set to $30 \mathrm{mV}$ (square) and to $50 \mathrm{mV}$ (diamond).

(a)

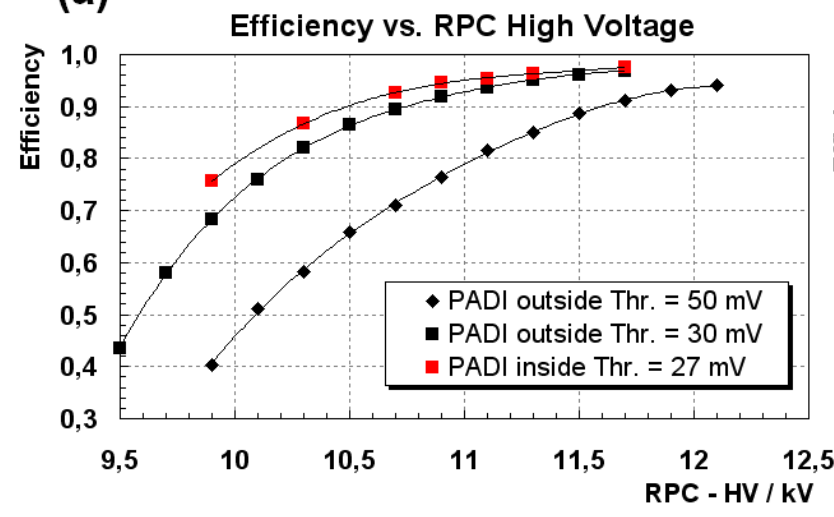

(b)

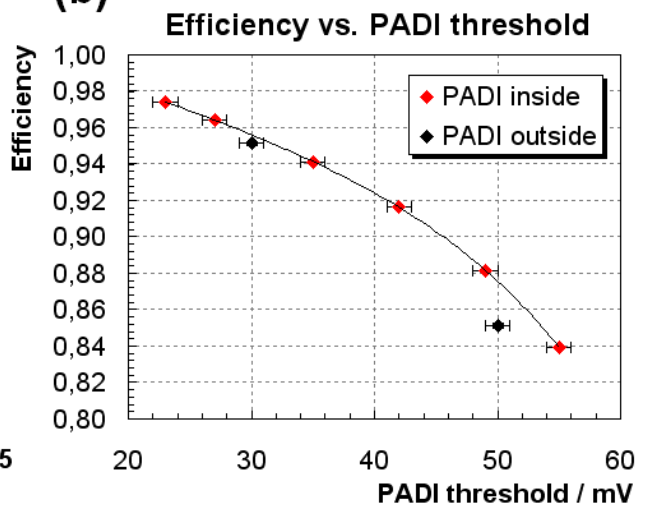

Figure 4: Efficiency as function (a) of applied RPC high voltage for preamplifier thresholds $\mathrm{U}_{t h r}=27$ $\mathrm{mV}$ (red squares), $\mathrm{U}_{t h r}=30 \mathrm{mV}$ (black squares) and $\mathrm{U}_{t h r}=30 \mathrm{mV}$ (black diamonds) (b) of preamplifier threshold. The red color represent data from the FEE connected directly to the RPC and black represents data from preamplifiers mounted outside the box.

This plot illustrates that the demanded efficiency of $95 \%$ is reachable for thresholds up to $30 \mathrm{mV}$ at the nominal working voltage of about $11.3 \mathrm{kV}$. Efficiency measurements done with the electronics 
mounted inside show slightly better results even if one scales them to the same threshold. A threshold scan for the inside mounted preamplifier is depicted in fig. 4b (red symbols). The two black data points stem from the PADI located outside. It turns out that the preamplifier mounted inside were much more stable in terms of pick-up noise from the environment. Therefore it was possible to lower the threshold almost by $10 \mathrm{mV}$ which is actually the bigger advantage. At a threshold of $23 \mathrm{mV}$ an efficiency above $97 \%$ was achieved. The applied voltage was $11.3 \mathrm{kV}$ and the particle flux below $500 \mathrm{~Hz} / \mathrm{cm}^{2}$. Figure 5 illustrate the efficiency as function of the flux measured at four different gas temperatures ( $\mathrm{HV}=11.5 \mathrm{kV}$, $\mathrm{U}_{\text {threshold }}=30 \mathrm{mV}$ ). The temperature is obtained by taking the mean of all 18 sensors. At room temperature $\left(27^{\circ} \mathrm{C}\right)$ already at $1 \mathrm{kHz} / \mathrm{cm}^{2}$ the efficiency starts to degrade and at $2 \mathrm{kHz} / \mathrm{cm}^{2}$ which can occur in the very innermost part of region A it drops to $85 \%$. Warming up the gas by 15 degree seems to be sufficient to obtain a fully efficient counter. By warming up to $50{ }^{\circ} \mathrm{C}$ still $90 \%$ efficiency is achievable for a particle flux of $15 \mathrm{kHz} / \mathrm{cm}^{2}$.

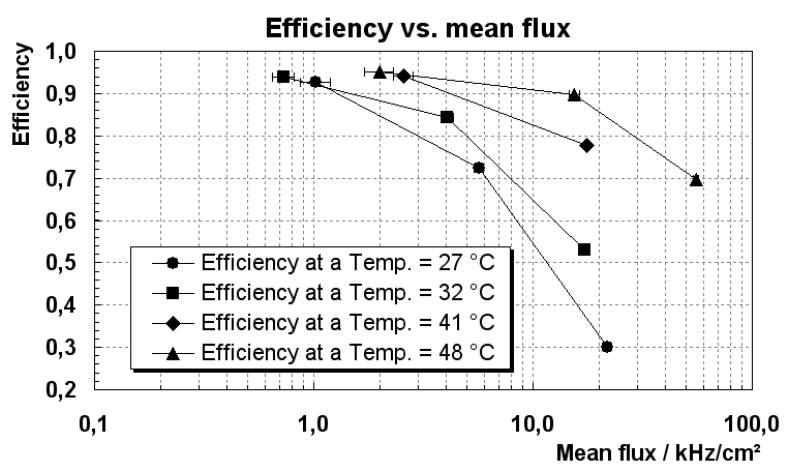

Figure 5: Efficiency as function of incident particle flux for the gas temperatures $\vartheta=27{ }^{\circ} \mathrm{C}$ (dots), $32{ }^{\circ} \mathrm{C}$ (diamonds), $41{ }^{\circ} \mathrm{C}$ (squares), $48^{\circ} \mathrm{C}$ (triangles).

\section{Time resolution}

The time resolution is the most critical and important parameter for region A. At low particle fluxes a time resolution of $51 \mathrm{ps}$ was measured taking the time difference between a reference counter developed in Bucharest [6] and the HD-counter assuming both have the same resolution (see fig. 6a). However this result was obtained taking only one strip from both counters into account. This measurement was performed at GSI at low particle flux $\left(<100 \mathrm{~Hz} / \mathrm{cm}^{2}\right)$ and a beam spot size of about $8 \mathrm{~cm}$ in diameter. Figure $6 \mathrm{~b}$ illustrate the time resolution as function of the incident proton flux for different temperatures measured at COSY. The beam spot size was estimated to be about $0.4 \mathrm{~cm}^{2}$. The rate of the plastic scintillator hits was recorded in the data. The QDC and time over threshold information of each contributing channel was used to correct the pulse height dependencies (walk) of the measured time difference between the average of the PMT and the RPC times. Additional corrections on the nonlinearities of the TDC were done in order to minimize the electronic time resolution. The time resolution is rising as expected with the flux but can be improved dramatically by warming. This can be seen at best from the data points between $15 \mathrm{kHz} / \mathrm{cm}^{2}$ and $20 \mathrm{kHz} / \mathrm{cm}^{2}$. This result agrees with the well known fact that a temperature increase of $26{ }^{\circ} \mathrm{C}$ improves the rate capability by one order of magnitude. Nevertheless a time resolution better than $70 \mathrm{ps}$ using the plastic scintillator as reference was not observed in any prototype. On the other hand the Bucharest group measured a time resolution in the order of $50 \mathrm{ps}$ of there counters using a second MRPC as 
reference which was connected to the same TDC [7]. That lead to the assumption that the TDC synchronization was not fully under control.

(a)

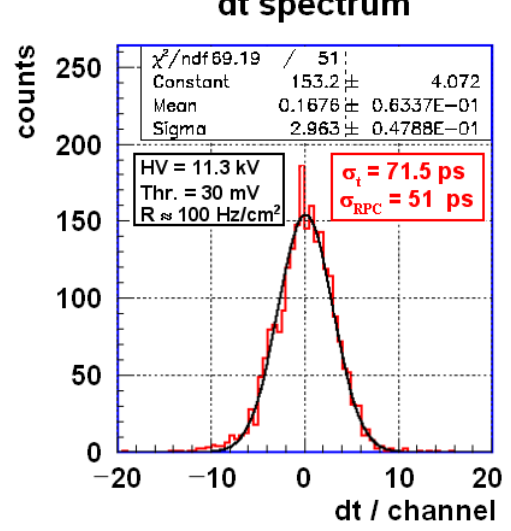

(b)

Time resolution vs. mean flux

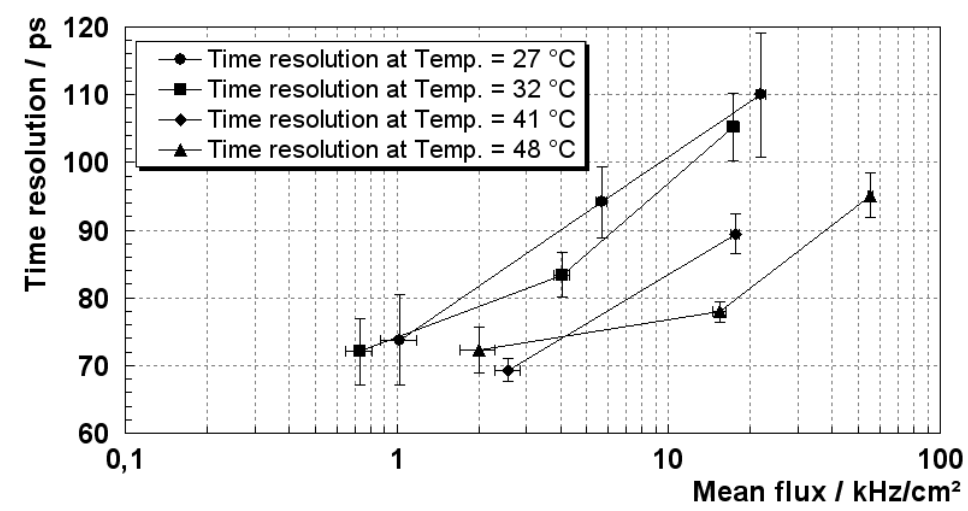

Figure 6: (a) Time difference dt between RPC to RPC (fore more details see text) (b) Time resolution as function of incident particle flux for the gas temperatures $\vartheta=27{ }^{\circ} \mathrm{C}$ (dots), $32{ }^{\circ} \mathrm{C}$ (diamonds), $41{ }^{\circ} \mathrm{C}$ (squares), $48^{\circ} \mathrm{C}$ (triangles).

\section{Mean cluster size}

The Mean Cluster Size MCS is defined as the average number of neighboring strips which fire simultaneously. Hence it is an important parameter in determining the effective counter granularity. At nominal working voltage $(11.3 \mathrm{kV})$ it is in the order of 1.3 strips and is directly connected with RPC-HV (0.04 per $100 \mathrm{~V} @ 11.3 \mathrm{kV})$ with a rising slope towers higher voltages. Since charge sharing over more strips is the cause of clusters bigger than 1 the mean cluster size depends strongly on the preamplifier threshold. The data show a logarithmic dependence of the mean cluster size vs. threshold. Also a logarithmic behavior is observed in the mean cluster size as function of the mean particle flux. The advantages in terms of efficiency and time resolution gained by warming is counterbalanced with the disadvantages resulting from the increased cluster size which induce not only a higher effective counter granularity and therefore a reduced double hit capability but also a higher data processing time.

\section{Summary and Conclusion}

In this proceeding we presented a Multistrip-MRPC prototype developed at Physikalisches Institut der Universität Heidelberg. This counter is designed for the low rate environment called region A for the CBM-Tof wall and it was tested in beams at two different locations with different conditions. Efficiency measurements show that the minimal goal of $95 \%$ was reached and in case of inside mounted electronics due to lower pickup noise (shielding) and therefore lower possible threshold settings even exceeded. The targeted counter time resolution of $50 \mathrm{ps}$ (needed in order to have the required $80 \mathrm{ps}$ system time resolution) is partially fulfilled although a full surface illumination test with realistic rates is needed to be done. We were able to show that increasing the gas temperature by $15^{\circ} \mathrm{C}$ is sufficient to be fully operational up to $2.5 \mathrm{kHz} / \mathrm{cm}^{2}$. Nevertheless 
higher temperatures lead to bigger cluster sizes and thereby to lower occupancy. It is also proven that in our setup mounting the preamplifier close to the readout electrode is the better choice. With the knowledge gained from this prototype we plan to produce a so-called full size demonstrator with about $50 \times 50 \mathrm{~cm}^{2}$.

\section{References}

[1] B.Friman, C. Höhne, J. Knoll, S. Leupold, J. Randrup, R. Rapp, P. Senger, The CBM Physics Book, Compressed Baryonic Matter in Laboratory Experiments, Lecture Notes in Physics, Vol. 814 st Edition (2011) [ISBN 978-3-642-13292-6]

[2] I. Deppner et al., Nucl. Instrum. Methods A 661 Sup. 1 (2012) 121

[3] S. A. Bass et al., Microscopic Models for Ultrarelativistic Heavy Ion Collisions, Prog. Part. Nucl. Phys. 41 (1998) 225-370

[4] I. Deppner at al, Characterization of FOPI narrow strip RPCs, CBM Progress Report (2008)

[5] M. Ciobanu et al. IEEE NSS Dresden (2008) 2018-2024

[6] M. Petriş et al. Nucl. Instrum. Methods A 661 Sup. 1 (2012) 129

[7] M. Petrovici, et al., High counting rate, differential, strip read-out, multi gap RPC, this proceeding 